\title{
SOUL: the Single conjugated adaptive Optics Upgrade for LBT
}

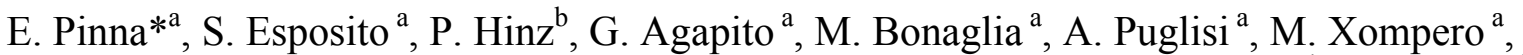

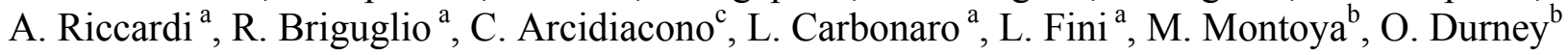 \\ ${ }^{a}$ INAF - Osservatorio Astrofisico di Arcetri, L.go E. Fermi 5, 50125 Firenze, Italy; \\ ${ }^{\mathrm{b}}$ Steward Observatory, University of Arizona, 933 North Cherry Avenue, Tucson, AZ, USA; \\ ${ }^{\mathrm{c}} \mathrm{INAF}$ - Osservatorio Astronomico di Bologna, Via Ranzani, 1, 40127 Bologna, Italy
}

\begin{abstract}
We present here SOUL: the Single conjugated adaptive Optics Upgrade for LBT. Soul will upgrade the wavefront sensors replacing the existing CCD detector with an EMCCD camera and the rest of the system in order to enable the closed loop operations at a faster cycle rate and with higher number of slopes. Thanks to reduced noise, higher number of pixel and framerate, we expect a gain (for a given SR) around 1.5-2 magnitudes at all wavelengths in the range 7.5 $<\mathrm{mR}$ $<18$. The correction at short wavelength will be greatly improved (SR $>70 \%$ in I-band and 0.6 asec seeing) and the sky coverage will be multiplied by a factor 5 at all galactic latitudes. Upgrading the SCAO systems at all the 4 focal stations, SOUL will provide these benefits in 2017 to the LBTI interferometer and in 2018 to the 2 LUCI NIR spectro-imagers. In the same year the SOUL correction will be exploited also by the new generation of LBT instruments: V-SHARK, SHARK-NIR and iLocater.
\end{abstract}

Keywords: Pyramid WFS, Adaptive Secondary, SCAO, eXAO, Large Binocular Telescope.

\section{INTRODUCTION}

SOUL: the Single conjugated adaptive Optics Upgrade for LBT [1] is an answer to the "2014 Call for Proposals for Instrument Upgrades and New Instruments" issued by LBTO. Currently, there are 4 SCAO systems [2] operating at LBT, all composed by an Adaptive Secondary Mirror [3] (ASM with 672 actuators) and a Pyramid Wavefront Sensor (PWFS with 30x30 sub-apertures). Starting the on-sky operations in 2010, these systems provided the first high contrast images ever on $8 \mathrm{~m}$-class telescopes in the NIR. The use of state-of-the-art devices is critical for the AO performances and, after about 10 years from the LBT SCAO design, the technological development made significant steps forward especially in the visible detector field. The main hardware upgrade that SOUL introduces is the replacement of the current wavefront sensor camera with an EMCCD one. The main benefits will be a shorter read-out time, a lower read-out noise and a larger number of pixels, allowing higher spatial sampling. Of course, other parts of the system require an upgrade in order to fully exploit these new features.

\section{THE UPGRADE}

\subsection{Wavefront sensor}

As said, the core of the upgrade is the implementation of a new generation visible detector for the wavefront sensing. Error budget computations and E2E simulations led us to the selection of the OCAM2k as new WFS detector. This camera is produced by First Light Imaging and is the latest version of the OCam [4]. It hosts an electron multiplied CCD chip (E2V CCD220) of 240x240 pixels of $24 \mu \mathrm{m}$ side. The typical performances of the camera show a RON of $0.37 \mathrm{e}^{-}$at $2.0 \mathrm{kHz}$ of framerate. In Table 1 we compare the main specifications of CCD39 (current WFS camera) and OCAM2k. Our numerical analysis identified the sampling of 40Sub-Apertures (SA) across the pupil as the best compromise between high correction at the bright end and performances in the faint one. The pupil sampling can be then reduced accordingly with the star flux to 20,13.3 and 10SA thanks to the CCD binning.

The 40SA pupil sampling will be achieved replacing the camera lens with a new one having a slight increased focal length (46mm vs. $36 \mathrm{~mm}$ ). The new lens, coupled with the existing double pyramid [5], will provide the required magnification (40 pix across) and distance (48 pix center-to-center) between the pupil images. In Figure 1 we show the optical configuration of the WFS board and in Figure 2 the pupil arrangement on the OCAM2k achieved with the new camera lens.

Adaptive Optics Systems V, edited by Enrico Marchetti, Laird M. Close, Jean-Pierre Véran, Proc. of SPIE Vol. 9909, 99093V (C) 2016 SPIE · CCC code: 0277-786X/16/\$18 - doi: 10.1117/12.2234444 
Table 1. The main technical features of the current WFS camera (CCD39) compared with those of the new one (OCAM2k)

\begin{tabular}{|c|c|c|}
\hline Camera/specification & CCD39 & Ocam2k \\
\hline Chip size & $80 \times 80$ & $240 \times 240$ \\
\hline Pixel side & 24 & 24 \\
\hline RON & $10.5 @ 1 \mathrm{kfps}$ & $0.37 @ 2 \mathrm{kfps}$ and $\mathrm{G}=400$ \\
\hline Excess noise & $\mathrm{NO}$ & $\sqrt{2}$ \\
\hline Max. framerate $\quad[\mathrm{fps}]$ & 1000 & $\begin{array}{ll}2000 & \text { (full frame) } \\
3625 & \text { (ROI 120x120) }\end{array}$ \\
\hline Min. read-out time $[\mathrm{ms}]$ & 0.95 & $\begin{array}{ll}0.24 & \text { (ROI 120x120) }\end{array}$ \\
\hline
\end{tabular}

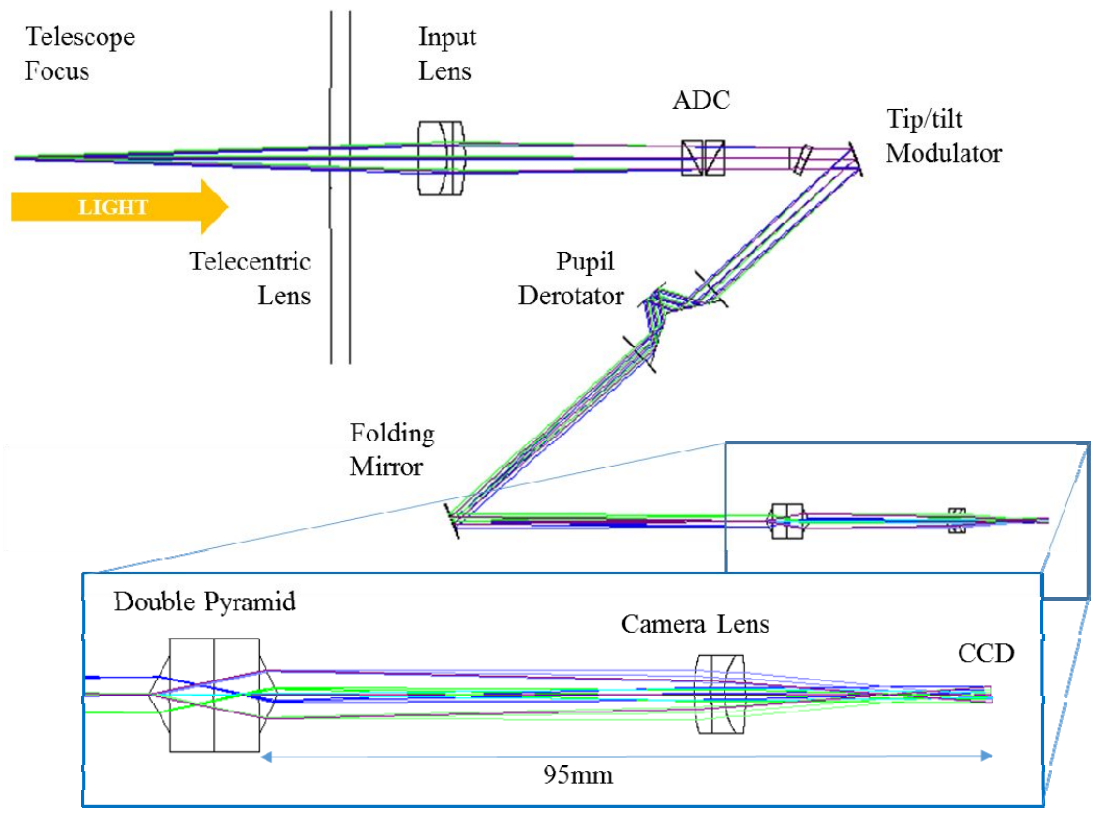

Figure 1. The optical layout of the WFS board of the FLAO system upgraded with the new camera lens (see zoom).

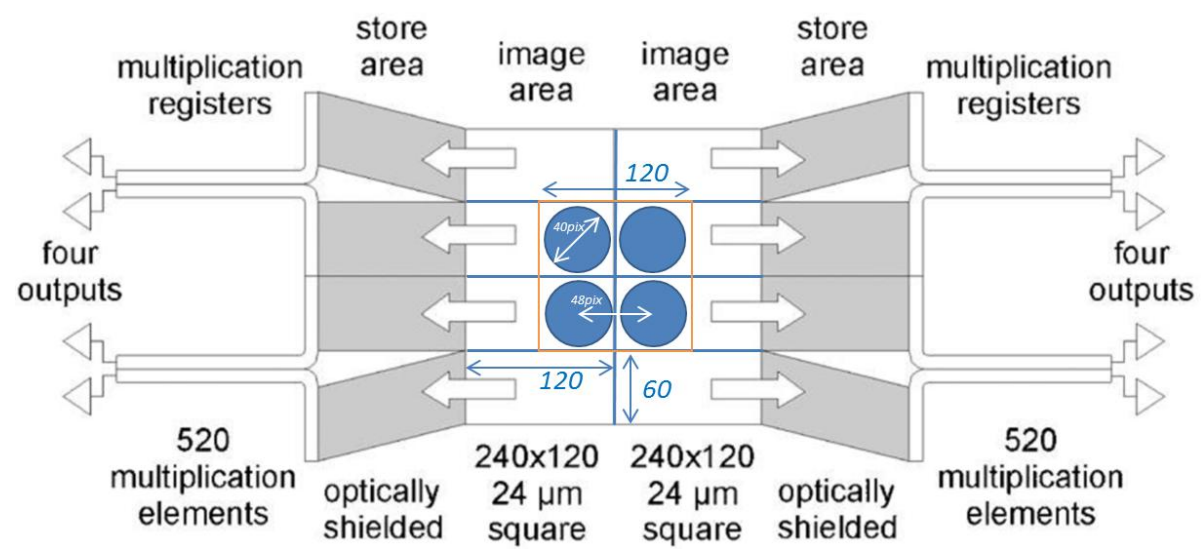

Figure 2. Schematic of the OCAM2k chip and read-out regions, together with arrangement of the 4 pupil images on a ROI of 120x120pixels. 
The LBTI and FLAO PWFSs are close to be identical and both adopt the tip-tilt modulation. This modulation allows the adjustment of the WFS sensitivity accordingly to the reference star magnitude and seeing conditions. The tiptilt modulation is provided by a flat mirror mounted on a piezo stage motor driving the mirror on a circular pattern at each CCD exposure frame. In order to allow close loop operations at $2.0 \mathrm{kHz}$, the tip-tilt motor shall be able to produce at the same frequency the desired circular pattern with the requested wavefront amplitude $( \pm 3 \lambda / \mathrm{D})$. Such requirement is beyond the performances of the device currently installed (PI S-325). During the prototyping work for the GMT-NGWS [6], we verified that the model PI-S330 equipped with high frequency electronics can provide the required performances. We plan to replace the driver electronics and we will evaluate via dedicated lab tests the needing of the piezo head replacement.

In Figure 3 we report two views of the mechanical layout of the FLAO WFS board after the SOUL upgrade.
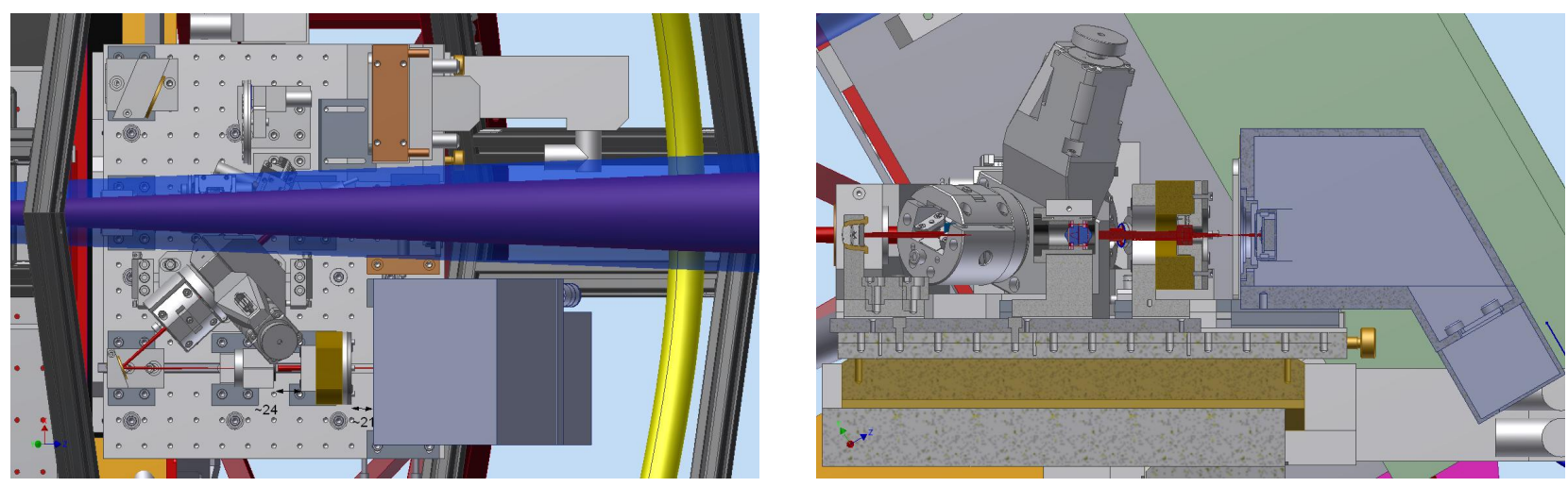

Figure 3. Mechanical layout of the FLAO WFS board after the SOUL upgrade. Left: top view showing the incoming f/15 beam from the telescope before the reflection on the instrument window. Right: side view showing the new camera (on the right) in its custom layout together with the new camera lens and the pyramid installed in their mounts.

Another component of the system that requires to be updated is the slope computer. This in order to work with a higher number of pixels $(\sim 5700)$, slopes $(\sim 2850)$ and at a faster framerate (up to 2.0kHz). Microgate designed the upgrade of the current slope computer enabling it to read the OCAM2k frames and provide the required slope computation within $0.17 \mathrm{~ms}$. The slope computation will be parallelized with the CCD readout (starting after the download of the first half of pixels), so that the contribution of the slope computation to the loop delay will be just of a few tens of microseconds. In order to achieve this performance, an extra DSP board will be added to the current slope computer and the firmware accordingly updated.

\subsection{Adaptive Secondary and Real Time Computer}

We performed some preliminary test on the FLAO system in order to assess the capabilities of the Adaptive Secondary Mirror (ASM) and all the real-time AO control to run at framerates higher than $1.0 \mathrm{kHz}$. As first, we tested the stability of the mirror itself and measured the step responses when a faster pre-shaping command is sent [7]. We have been able to test frequency up to $1.8 \mathrm{kHz}$ and the mirror showed to be stable and the modal step responses had a maximum overshoot of about $15 \%$ in the worst cases. Finally, we tested the entire AO loop using the calibration source and the current FLAO wavefront sensor. Binning the CCD39 we reached the frequency of $1.8 \mathrm{kHz}$ and the AO loop has been successfully closed at this framerate. This result proofs that no modification to the ASM hardware and firmware are required in order to reach the framerate baseline $(1.5 \mathrm{kHz})$. Due to the tight project schedule (sect. 4 ), we are currently planning to not implement the ASM firmware upgrade foreseen in the design phase.

The analysis performed by Microgate in the design phase showed that the current real-time communication bandwidth and computational power can already perform the wavefront reconstruction with 2750 slopes at $2.0 \mathrm{kHz}$ introducing a total delay of just $0.27 \mathrm{~ms}$. Moreover, ASM memory can already deal with the increased number of slopes without any modification. We conclude that the ASM itself and the RTC hosted on its own electronics will not require any upgrade in order to meet the SOUL requirements. 


\subsection{Control software}

The implementation of SOUL requires an upgrade of the AO control SoftWare (SW). The WFS control SW will be modified in order to cope with the new camera and the new slope computer. Then, other elements will require modifications in order to cope with the higher number of slopes. In particular, we refer to: the ASM control, the Arbitrators, the Master diagnostics and the elaboration library that is used to reduce the diagnostic data collected in the AO operations. A general requirement for the SW upgrade is to maintain the full compatibility with the WFSs of the other systems like ARGOS, LINC-NIRVANA and FLAO not yet upgraded.

Being SOUL an upgrade, the software will not be completely tested on the hardware before the final installation. In order to debug and deeply test the mentioned modifications, we will develop a simulation tool emulating the data streaming usually produced by the real hardware.

\subsection{AO loop delay}

In this section, we evaluate the delay offered by SOUL compared to the one of the current systems. In this analysis, we considered the baseline for the system framerate $(1.5 \mathrm{kHz})$ and the current value $(1.0 \mathrm{~ms})$ for the deformable mirror settling time. Figure 4 shows the delay diagram for SOUL and FLAO ${ }^{1}$. The computation shows a total delay for SOUL of $1.79 \mathrm{~ms}$ vs. $2.74 \mathrm{~ms}$ for FLAO. The main improvements in term of delay are given by the higher framerate (from 1.0 to $1.5 \mathrm{kHz}$ ), reducing integration time and hold, and the lower read-out time (from $0.95 \mathrm{~ms}$ to the $0.24 \mathrm{~ms}$ of the OCAM $2 \mathrm{k}$ when a sub-frame of 120X120pix is read).

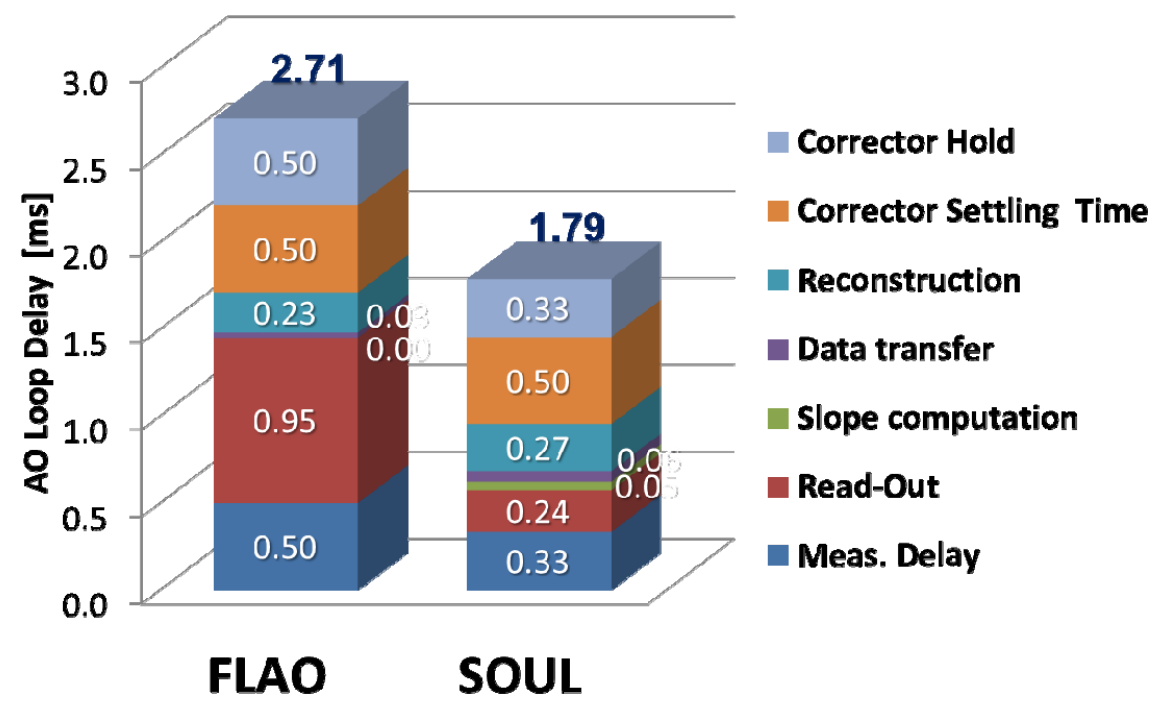

Figure 4. The diagram shows the total and the components of the AO loop delay in the case of SOUL (left) and FLAO (right).

\section{NUMERICAL SIMULATIONS}

We developed a semi-analytical model for the AO error budget estimation. This allowed us to completely explore the system parameters, identifying the best configuration in terms of residual wavefront as function of the reference star brightness. We did this optimization considering a single seeing condition $(0.8 \mathrm{arcsec})$, then we verified the performance estimations via E2E simulation for different seeing values and, finally, we estimated the improvement in sky coverage.

\footnotetext{
${ }^{1}$ Here FLAO is used as reference of the SCAO system before the SOUL upgrade. In terms of AO control FLAO and LBTI systems are fully equivalent.
} 


\subsection{Simulations parameters}

We summarize here the parameters we used for the error budget and E2E simulations.

- Telescope:

diameter $=8.22 \mathrm{~m}$

central linear obstruction $=11.1 \%$

- Observing conditions:

atmospheric turbulence: $0.6,0.8$ and 1.0 arcsec seeing, $\mathrm{L} 0=40 \mathrm{~m}$, vertical distribution see Table 2

no sky background (in gray nights negligible impact, verified)

- Vibrations:

where vibrations are considered we refer to a real "bad-vibration" spectrum measured on FLAO with open loop PSF jitter 63mas RMS

- Optical transmission:

total transmission (atmosphere, optics and quantum efficiency): 0.29 .

- Guide Star:

$\mathrm{R}$ magnitude scaled considering the 0 -magnitude $=1.99 \times 1010 \mathrm{ph} / \mathrm{m} 2 / \mathrm{s}$

- Deformable mirror:

real influence functions measured on the adaptive secondary mirror of LBT

- WFS detector:

OCAM2k (CCD220)

RON values (EMCCD gain $=1)$ : $150 \mathrm{e}-/$ pixel/frame.

Excess noise simulated using proper statistics of electro-magnifying process

Dark current: $\left(0.444 / f_{s}+0.00556\right) b^{2}$ e-/pixel/s (fs = sampling frequency, $\mathrm{b}=$ binning).

$\mathrm{EMCCD}$ gain $=400$

- WFS optics:

40x40 sub-aperture Pyramid WFS

FoV: 2.1 arcsec diameter

Central Wavelength: $\lambda_{\mathrm{wfs}}=750 \mathrm{~nm}$

Bandwidth: $600-900 \mathrm{~nm}$

- AO RTC:

Max frame frequency: $1.5 \mathrm{kHz}$.

Modal control with static modal gains

Modal base: Zernike Tip, Tilt, Focus and higher order 627 Karhunen-Loève modes fitted on the ASM

influence functions.

Temporal controller: simple integrator

- Total time delay: $1.79 \mathrm{~ms}$ (see sect. 2.4 )

Table 2. The main parameters of the two turbulence layer considered for the numerical simulations.

\begin{tabular}{|l|l|l|}
\hline Layer number & $\mathbf{1}$ & $\mathbf{2}$ \\
\hline Height from ground $[\mathrm{m}]$ & 0 & 6000 \\
\hline Cn2 fraction & 0.6 & 0.4 \\
\hline Speed $[\mathrm{m} / \mathrm{s}]$ & 15 & 18 \\
\hline
\end{tabular}

\subsection{Error budget analysis}

The error budget elements we considered for the SOUL parameter optimization are:

- Temporal error (A)

- Fitting error (A)

- Measurement Noise $(\mathrm{N})$

- $\quad$ Aliasing $(\mathrm{N})$ 
where the terms marked with "A" are represented by an analytical model, while those marked with "N" are taken into account numerically ${ }^{2}$. We evaluated the impact of these terms considering their propagation through the closed loop transfer function (A) including the effect of the pyramid optical gain in partial correction (N).

We optimized the AO parameters for each of the considered reference star magnitude $\left(7.5<\mathrm{m}_{\mathrm{R}}<18.5\right.$ at 1 magnitude steps). The considered parameters are: pupil sampling (CCD binning), number or corrected modes, loop framerate and integrator gain (here a single value is considered for all modes). We performed this optimization for both FLAO and SOUL cases, considering a seeing of $0.8^{\prime \prime}, \mathrm{L}_{0}=40 \mathrm{~m}$ and a wind speed of $16 \mathrm{~m} / \mathrm{s}$. Exploring the complete parameter space, we identified the best configuration as the one providing the lower wavefront residuals. In the plots of Figure 5 we report the optimized parameters for the two cases as function of the reference star magnitude. We can see how the SOUL improvements shift the optimal point of work to faster framerates, higher pupil sampling and higher number of corrected modes. This translates in to lower wavefront residuals at all the considered reference star brightness. In Figure 6 we report the residual wavefront variance estimated by the error budget together with the breakdown in the different components. These charts show the overall SOUL benefits at all magnitudes. In particular, the aliasing is reduced to a negligible value thanks to the pupil oversampling. Noise and time errors directly benefit of the reduced RON and loop delay respectively. Thanks to the higher number of controlled modes, the fitting error is strongly reduced for $m_{R}>9.5$.

Loop framerate

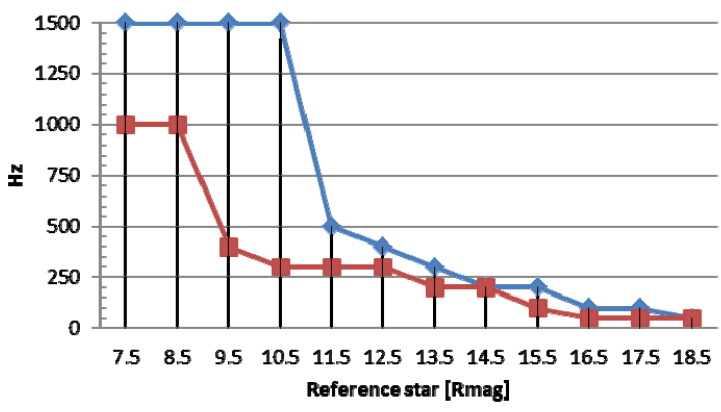

Corrected modes

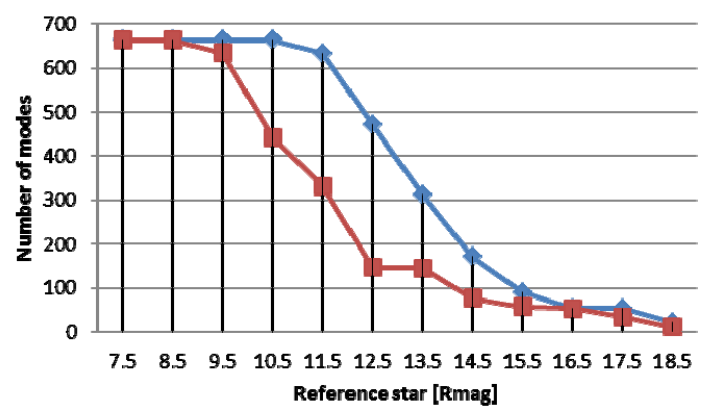

Pupil sampling

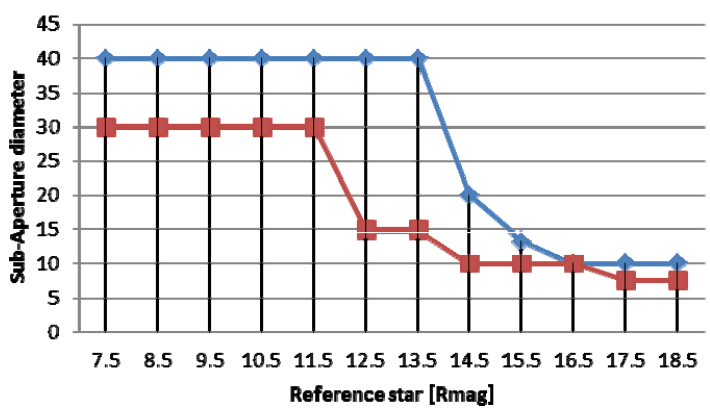


gain of 1 to 2 magnitudes to obtain the same level of correction obtained by FLAO. When bad vibrations are considered (dashed line) the SOUL benefit increases up to 3 magnitudes.
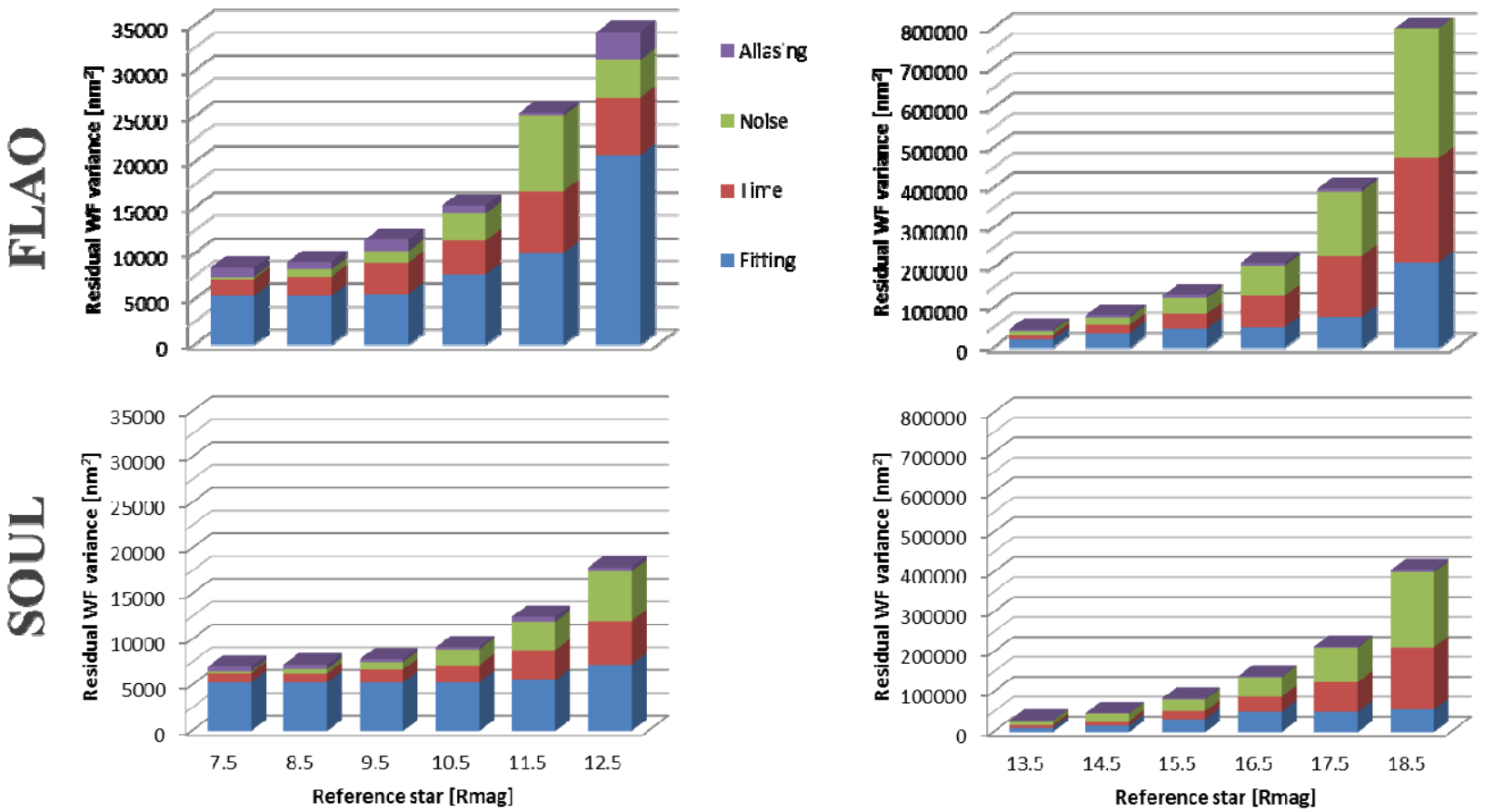

Figure 6. The residual wavefront variance as estimated via error budget. We compare here the FLAO (top) and SOUL (bottom) values as a function of the reference star magnitude. The different colors represent the different error terms contributions (aliasing, noise, time and fitting). All the values are obtained for a seeing of 0.8 asec.

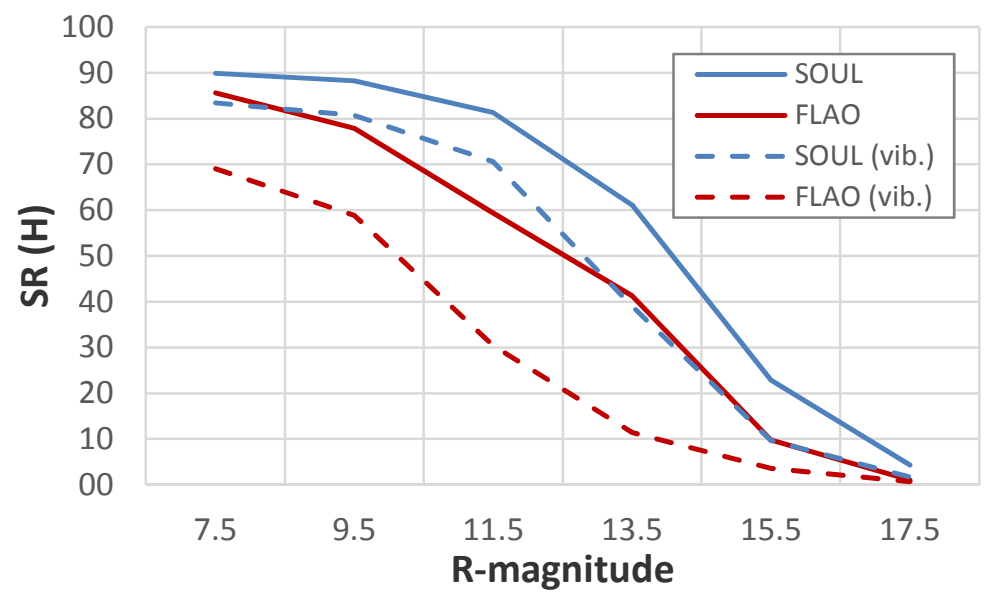

Figure 7. Strehl ratio values obtained at $1650 \mathrm{~nm}$ via E2E simulation for the case of SOUL and FLAO. The dashed line represents the case considering strong telescope vibrations. 

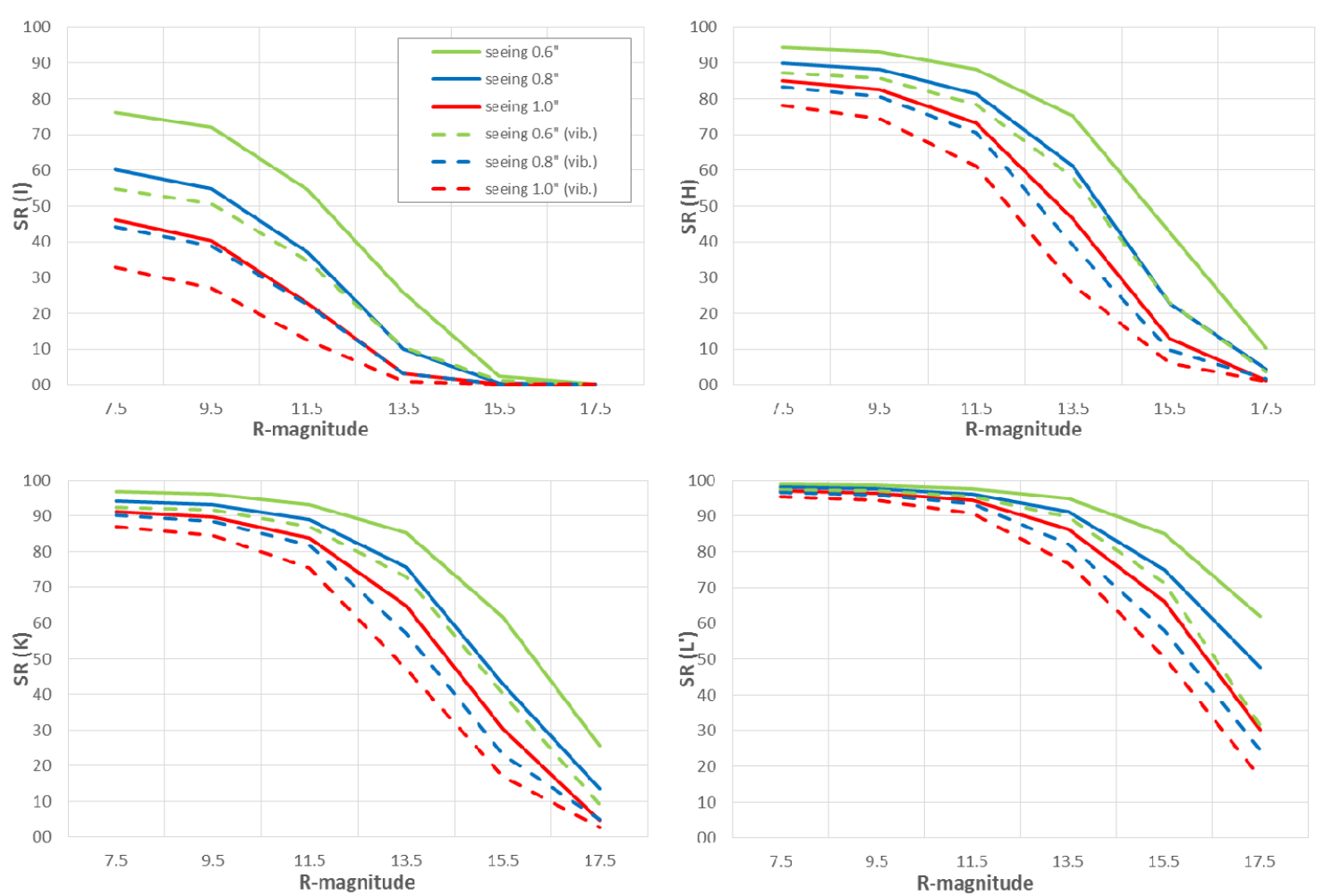

Figure 8. Strehl ratio values computed via E2E simulations for SOUL. The colors correspond to different seeing values, while the dashed line represents the case considering strong telescope vibrations. Each plot corresponds to different scientific bands I, H, L' and $\mathrm{K}$ starting from top left and following clockwise.

We estimated the SR values obtained on-axis by SOUL at different bands and seeing conditions and we report a sample of these results in Figure 8. We want to remark here the high SR values foreseen at 750nm (I-band) for $\mathrm{m}_{\mathrm{R}}<11.5$ and for $m_{R}=17.5$ at $2200 \mathrm{~nm}$ (K-band). The first one will open the visible AO science to a much greater number of objects than currently possible [9][10]; the second one will allow NGSAO correction for extragalactic science as we will see here below.

We performed the sky coverage estimations for both cases FLAO and SOUL, considering vibrations, a 4 layer atmospheric profile [11][12](see Table 3) and estimating the off-axis SR values via E2E simulations. We applied the results to a sample of $1.9 \cdot 10^{5}$ real sky positions evaluating the best wavefront correction achievable with the available reference stars. In Figure 9 we compare the sky coverage obtained with FLAO and SOUL as a function of the galactic latitude. Setting the SR limit to $20 \%$ we found in H-band an increase of 5 times the FLAO coverage at all latitudes, while, in K-band, SOUL brings the value to almost $20 \%$ at the galactic pole.

Table 3. The parameters of the atmospheric model used for the sky coverage computation.

\begin{tabular}{|c|c|c|c|c|}
\hline \multicolumn{5}{|c|}{ Turbulence } \\
\hline Seeing [arcsec] & \multicolumn{4}{|c|}{0.875} \\
\hline Outer scale $\left(\mathrm{L}_{0}\right)[\mathrm{m}]$ & \multicolumn{4}{|c|}{40} \\
\hline$\tau_{0}[\mathrm{~ms}]$ & \multicolumn{4}{|c|}{5.0} \\
\hline \multicolumn{5}{|c|}{ Layers } \\
\hline Altitude [m] & 119 & 837 & 3025 & 12780 \\
\hline Wind speed $[\mathbf{m} / \mathbf{s}]$ & 2.0 & 4.0 & 6.0 & 25.0 \\
\hline Relative $C_{n}^{2}$ & 0.70 & 0.06 & 0.14 & 0.10 \\
\hline
\end{tabular}



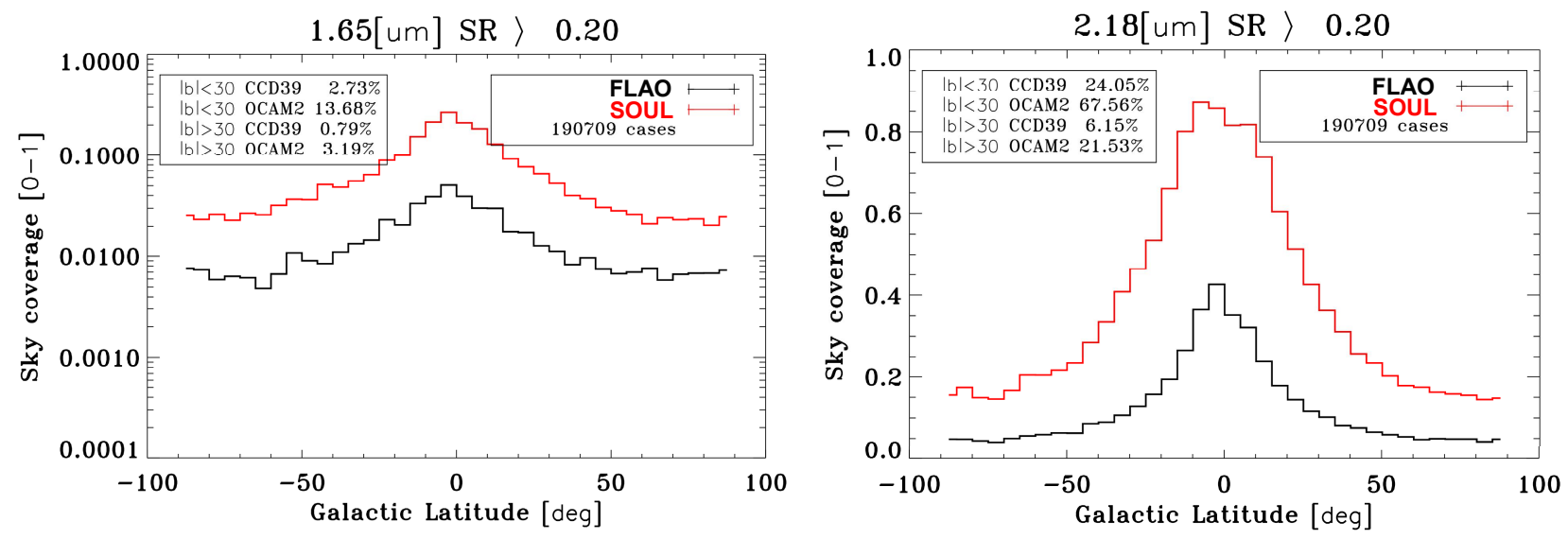

Figure 9. Comparison of the sky coverage obtained for SOUL and FLAO. Left: in H band we gain a factor 5 at all galactic latitudes. Right: in $\mathrm{K}$ band we reach high values even at the galactic pole.

\section{PROJECT SCHEDULE}

The schedule of the project is driven by the fact that SOUL is an upgrade to systems currently operating at the telescope. The implementation must be executed avoiding a significant impact on the telescope observing schedule. The temporal window available is then reduced to the so-called "LBT summer shutdown", when the telescope stops the science operations because of the monsoon season. This period lasts about 2 months and we plan to use 2 of them: the next one (summer 2017) for the integration of the systems at the LBTI [13] focal stations and the following one (summer 2018) for those feeding LUCI1[14] and LUCI2. The SOUL team together with LBTO selected LBTI as first system for the upgrade because of new instruments SHARK-NIR [15] and V-SHARK [16] that will be integrated at these focal stations in 2018. Both of them rely on the SOUL performances for the achievement of their scientific goals.

SOUL successfully passed its design review in Tucson at the end of March 2016 and just started the procurement phase. The contract with First Light for the OCAM2k cameras has been placed and we expect to receive the detectors for the LBTI upgrade at beginning of October 2016. In June 2017 we plan to ship the hardware for LBTI in Arizona and start the integration on the wavefront sensors. After the summer shutdown, we will start the on-sky commissioning. The same timeline will be repeated one year later for the upgrade of the 2 FLAO systems, as reported in the schedule of Figure 10.

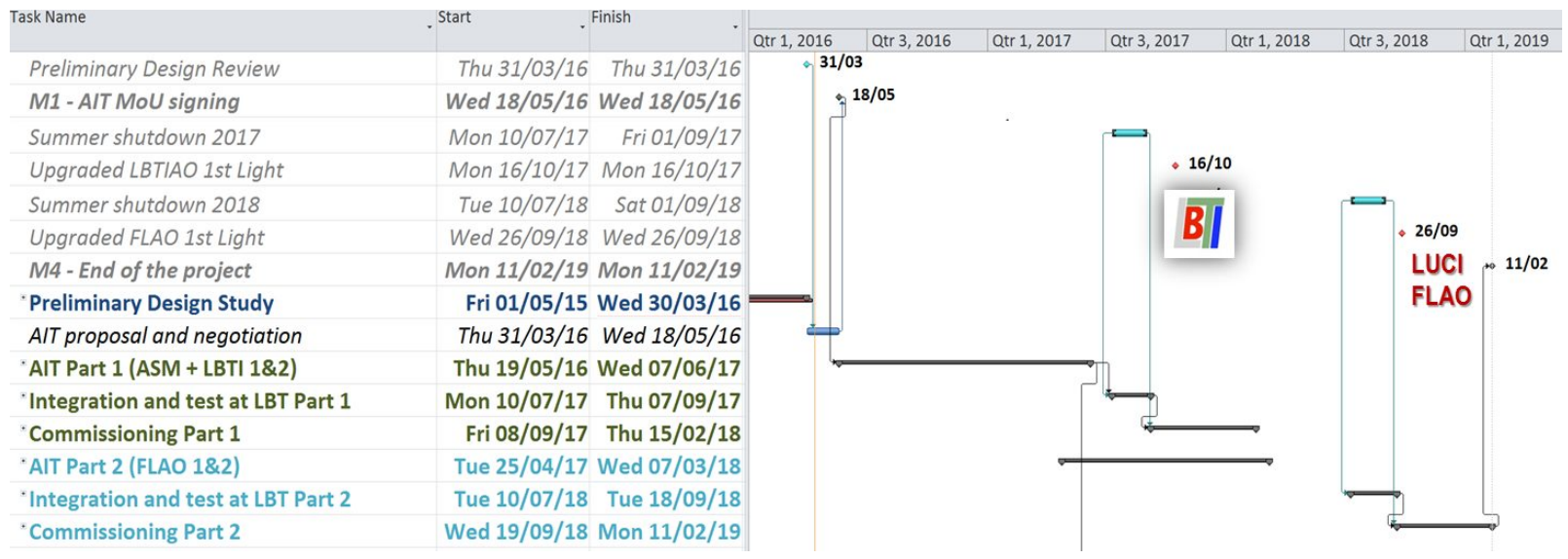

Figure 10. The Gantt chart of the SOUL project together with the main milestones. 


\section{CONCLUSIONS}

SOUL will upgrade the current SCAO systems at the LBT replacing the current wavefront sensor camera with an OCAM $2 \mathrm{k}$. This will allow to oversample the pupil, reduce the RON below $1 \mathrm{e}^{-}$and run the system at least at $1.5 \mathrm{kHz}$. In order to reach these new specifications, we will replace the WFS camera lens, the slope computer and refurbish the tiptilt modulator. The corrector (adaptive secondary) and the real-time computer will not require any modification.

The error budget analysis showed that all the error terms at all reference star magnitudes will be significantly reduced by the upgrade. The aliasing is reduced due to the pupil oversampling; the noise error benefits of the low RON; the time error benefits of the reduced delay and the faster frame-rate allowed by the low RON; finally, the fitting error is reduced at fainter magnitude due to the higher number of controlled modes. The overall improvement of SOUL can be summarized in a gain of 1 to 2 magnitudes in references star brightness over the entire range $7.5<\mathrm{m}_{R}<17.5$. This translates in shifting the high contrast regime in the NIR from $\mathrm{m}_{\mathrm{R}}=9.5$ to 11.5 and increasing the sky coverage in H-band of about a factor 5 and allowing in K-band to have a relevant fraction of sky covered even at the galactic pole.

SOUL will upgrade a total of 4 AO systems located at different focal stations. The 2 of LBTI will have the first light in the fall 2017, being ready for the integration of the high contrast imager and coronograph SHARK-NIR and VSHARK. The same AO system will also feed the LBT planet finder called iLocator [17]. In the fall 2018 is planned the first light of the upgrade for the 2 FLAO systems feeding the NIR spectro-imagers LUCI1 and LUCI2.

\section{REFERENCES}

[1] Hill, J. M. et al., "The Large Binocular Telescope," Proc. SPIE 7012, (2008).

[2] Esposito, S. et al., "Natural guide star adaptive optics systems at LBT: FLAO commissioning and science operations status," Proc. SPIE 8447, 84470U-1 (2012)

[3] Riccardi, A. Et al., "Adaptive secondary mirrors for the Large binocular telescope," in Proc. SPIE 5169, pp. 159-168 (2003).

[4] Feautrier, P., et al., "OCam with CCD220, the Fastest and Most Sensitive Camera to Date for AO Wavefront Sensing," PASP 123, Issue 901, pp. 263-274 (2011).

[5] Tozzi, A. et al., "The double pyramid wavefront sensor for LBT," Proc. SPIE 7015, article id. 701558, 9 pp. (2008).

[6] Pinna, E. et al, "Design and numerical simulations of the GMT Natural Guide star WFS," Proc. SPIE 9148, id. 91482M 15 pp. (2014).

[7] Riccardi, A. et al., "The adaptive secondary mirror for the Large Binocular Telescope: results of acceptance laboratory test," Proc. SPIE 7015, article id. 701512, 9 pp. (2008).

[8] Agapito, G. et al., "PASSATA: object oriented numerical simulation software for adaptive optics," Proc. SPIE 9909, paper 314 in these proceedings (2016).

[9] Morzinski, K. et al, "MagAO: Status and Science," Proc. SPIE 9909-01 (2016).

[10] Fusco, T. et al., "SPHERE on-sky results: final performance, lesson learned, and possible upgrades," Proc. SPIE 9909, paper 32 in these proceedings (2016).

[11] Masciadri, E. et al., "Optical turbulence vertical distribution with standard and high resolution at Mt Graham," MNRAS404, 144 (2010).

[12] Hagelin, S. et al., "Wind speed vertical distribution at Mt Graham," MNRAS407, 2230 (2010).

[13] Hinz, P., "First AO-corrected interferometry with LBTI: steps towards routine coherent imaging observations," Proc. SPIE 8455, 84450U (2012).

[14] Seifert, W. et al., "LUCIFER: a multi-mode NIR instrument for the LBT," Proc. SPIE, 4841, 962-973 (2003).

[15] Farinato, J. et al., "SHARK-NIR: from K-band to a key instrument, a status update," Proc. SPIE 9908, paper 116 (2016).

[16] Pedichini, F. et al., "The V-SHARK high contrast imager at LBT," Proc. SPIE 9908, paper -1163 (2016).

[17] Crepp, J. et al., "iLocater: An AO-fed Doppler spectrometer for the Large Binocular Telescope," Proc. SPIE 9908, paper 46 (2016). 